\title{
Effect of submaximal contraction intensity in contract-relax proprioceptive neuromuscular facilitation stretching
}

\section{J B Feland, H N Marin}

Br J Sports Med 2004;38:e18 (http://www.bjsportmed.com/cgi/content/full/38/4/e18). doi: 10.1136/bjsm.2003.010967

\begin{abstract}
Objective: To determine if submaximal contractions used in contract-relax proprioceptive neuromuscular facilitation (CRPNF) stretching of the hamstrings yield comparable gains in hamstring flexibility to maximal voluntary isometric contractions (MVICs).

Method: Randomised controlled trial. A convenience sample of 72 male subjects aged 18-27 was used. Subjects qualified by demonstrating tight hamstrings, defined as the inability to reach $70^{\circ}$ of hip flexion during a straight leg raise. Sixty subjects were randomly assigned to one of three treatment groups: $1,20 \%$ of MVIC; $2,60 \%$ of MVIC; $3,100 \%$ MVIC. Twelve subjects were randomly assigned to a control group (no stretching). Subjects in groups 1-3 performed three separate six second CRPNF stretches at the respective intensity with a 10 second rest between contractions, once a day for five days. Goniometric measurements of hamstring flexibility using a lying passive knee extension test were made before and after the stretching period to determine flexibility changes.

Results: Paired $t$ tests showed a significant change in flexibility for all treatment groups. A comparison of least squares means showed that there was no difference in flexibility gains between the treatment groups, but all treatment groups had significantly greater flexibility than the control group.

Conclusion: CRPNF stretching using submaximal contractions is just as beneficial at improving hamstring flexibility as maximal contractions, and may reduce the risk of injury associated with PNF stretching.
\end{abstract}

$\mathrm{F}$ lexibility is considered to be a valuable component of athletic performance and injury reduction. Several stretching methods, including static, ballistic, and proprioceptive neuromuscular facilitation (PNF), have been shown to increase flexibility, ${ }^{1-5}$ but the research is divided on which technique is most effective. PNF stretching has been reported to be more effective at improving range of motion than static or ballistic techniques. ${ }^{256}$ There are many variations of PNF stretching. The contract-relax (CR) method is a technique that uses a maximum voluntary isometric muscle contraction (MVIC) followed by relaxation. It has been shown that the most beneficial PNF contraction duration is $3-10$ seconds, with six seconds being preferred..$^{8}$

The correct intensity of a stretch has not been well defined, and very few studies have used different intensities in static ${ }^{10}$ and $\mathrm{PNF}^{8} 9$ stretching protocols. Contraction intensities in PNF stretching as low as $50 \%$ have been reported to produce similar flexibility gains to MVICs, ${ }^{9}$ although the primary purpose of that article was to show alterations in blood pressure. Submaximal contraction intensities could also reduce the risk of contraction induced injuries and delayed onset muscle soreness. To date, there are no studies on the effect of CRPNF stretching at lower than $50 \%$. Therefore, the purpose of this study was to determine the effect of varying intensities (20-100\%) of contraction used in CRPNF stretching on improving flexibility of the hamstring muscle group.

\section{METHODS}

This was a randomised controlled clinical trial in which 72 healthy college age men (mean (SD) age 22.6 (2.03) years) qualified to participate by exhibiting tight hamstrings (defined as the inability to reach $70^{\circ}$ hip flexion in a straight leg raise). We obtained approval from the institutional review board to use human subjects. All subjects participated in a training session on a Biodex System-3 isokinetic machine one week before actual testing to determine MVIC in a stretch position. Participants sat upright at $90^{\circ}$ hip flexion. Only the right leg was tested. With the right leg restrained at the midthigh and ankle, a tester passively moved the lower leg through knee extension until the stretch in the hamstrings began to feel "uncomfortable" to the subject. The isokinetic arm was locked in position, and the subject performed a maximal isometric contraction with the hamstring muscles for six seconds, followed by 10 seconds of relaxation. During the 10 seconds of relaxation, the tester slowly extended the subject's leg further until the same level of discomfort was felt. If the subject still considered the stretch to be uncomfortable, it was kept at the previous position. The subject then performed two more six second maximal contractions (for a total of three contractions) with 10 second relaxation periods in between.

Each subject's maximum contraction was calculated by taking an average of the three MVIC trials. Sixty subjects were then randomly assigned to one of three test groups: 1, $20 \%$ of MVIC; $2,60 \%$ of MVIC; 3, $100 \%$ MVIC. Twelve subjects were assigned to a control group (no stretching). The subjects started stretching one week after the training session. They were tested each day for five days.

Each subject's hamstring flexibility was measured twice a day, once before and once after stretching using a 12 inch goniometer. Participants laid supine with their left leg straight (being held to the table by an assistant), and their right leg at $90^{\circ}$ hip flexion. The right lower leg was then passively extended to the point of initial resistance, and measured. The same tester took all flexibility measurements, but an assistant recorded the scores so the tester was blinded to previous flexibility measurements of each subject.

After recording of initial flexibility levels, the subject then performed three trials on the Biodex as previously explained. All subjects contracted for six seconds followed by 10 seconds of relaxation and further extension. The only varying factor was the intensity of contraction, whether it was $20 \%$, $60 \%$, or $100 \%$ MVIC. Contraction torque was displayed

Abbreviations: $\mathrm{CR}$, contract-relax; $\mathrm{MVIC}$, maximum voluntary isometric muscle contraction; PNF, proprioceptive neuromuscular facilitation 
Table 1 Paired samples statistical analysis of change in flexibility over five days

\begin{tabular}{lllll}
\hline Group & df & $\begin{array}{l}\text { Mean } \\
\text { change }\end{array}\left(^{\circ}\right)$ & SD & p Value \\
\hline $1(20 \%)$ & 17 & 5.00 & 4.83 & 0.0001 \\
$2(60 \%)$ & 16 & 4.47 & 6.58 & 0.013 \\
$3(100 \%)$ & 14 & 5.13 & 5.11 & 0.002 \\
$4($ control) & 11 & 0.33 & 0.88 & 0.220 \\
\hline
\end{tabular}

visually as bar-type graph on the computer monitor to allow the subject to visually maintain a $20 \%$ or $60 \%$ contraction for each six second repetition. The 12 control subjects (group 4) were also measured twice with about five minutes between measurements to simulate the time it took to set up and stretch the subjects in the intervention groups.

\section{RESULTS}

All data were analysed in SPSS version 7.5. As each subject served as his own control, paired $t$ tests were generated to determine if a significant change in flexibility occurred within groups by comparing the flexibility measurement before the test on Monday with the flexibility measurement on Friday. Analysis of variance showed insignificant $(\mathrm{p}=$ 0.06) differences between treatment groups.

Subjects were disqualified from participation after missing one day of testing. This gave a final sample size of 18 in group $1(20 \%), 17$ in group $2(60 \%), 15$ in group $3(100 \%)$, and 12 in group 4 (control). The mean age of the participants was 22.6 years (range 18-27). The paired $t$ test showed that groups 1 , 2 , and 3 exhibited a significant change in flexibility, whereas group 4 did not significantly change (table 1).

\section{DISCUSSION}

It has long been standard to perform a maximal contraction in PNF techniques. However, maximal contractions are intense enough to produce symptoms of delayed onset muscle soreness and may increase the risk of injury. The results of this pilot study suggest that contractions at $20 \%$ and $60 \%$ MVIC are just as effective as 100\% MVIC during CRPNF hamstring stretching. The results also verify that all interventions improved flexibility more than no stretching (control group). Although the maximum contraction group showed greatest improvements overall, it averaged just $0.13^{\circ}$ greater flexibility than the $20 \%$ group, which, in our opinion, is not clinically significant.

One other submaximal PNF study ${ }^{8}$ suggested that PNF stretching should be submaximal ( $75 \%$ in their study) and progressive. Schmitt et $a l^{8}$ also proposed that soft tissue length may be neurologically "reset" through stretching, rather than permanent deformation to more resistive tissues, whereas Magnusson et al ${ }^{11}$ suggest that PNF stretching simply alters stretch perception. The results of our study, as with Schmitt et al, lead to further questions about the role of the muscle spindle and Golgi tendon organ response to submaximal contractions.

Whether submaximal contractions less than $20 \%$ would elicit a neurological response great enough to cause similar changes is not known. Future studies should try to determine an intensity threshold, as well as the effect of different contraction durations at a submaximal level.

\section{CONCLUSIONS}

The use of submaximal contraction intensities of $20 \%$ and $60 \%$ MVIC in CRPNF stretching of the hamstrings yields comparable gains in flexibility to $100 \%$ MVIC. The benefit is to make the stretch more comfortable and to decrease the risk of contraction induced injury. The exact mechanism behind these results is unclear, and future research should focus on finding neurophysiological and anatomical explanations.

\section{Authors' affiliations \\ J B Feland, H N Marin, Brigham Young University, Provo, UT 84602, USA}

Correspondence to: Dr Feland, Brigham Young University, RB-120A, Provo, UT 84602, USA; brent_feland@byu.edu

Accepted 6 January 2004

\section{REFERENCES}

1 Sady SP, Wortman M, Blanke D. Flexibility training: ballistic, static or proprioceptive neuromuscular facilitation? Arch Phys Med Rehabil 1982;63:261-3.

2 Wallin D, Ekblom B, Grahn R, et al. Improvement of muscle flexibility. A comparison between two techniques. Am J Sports Med 1985;13:263-8.

3 Bandy WD, Irion JM, Briggler $M$. The effect of time and frequency of static stretching on flexibility of the hamstring muscles. Phys Ther 1997;77:1090-6.

4 Lucas RC, Koslow R. Comparative study of static, dynamic, and proprioceptive neuromuscular facilitation stretching techniques on flexibility. Percept Mot Skills 1984;58:615-18.

5 Etnyre BR, Abraham LD. Gains in range of ankle dorsiflexion using three popular stretching techniques. Am J Phys Med 1986;65:189-96.

6 Funk DC, Swank AM, Mikla BM, et al. Impact of prior exercise on hamstring flexibility: a comparison of proprioceptive neuromuscular facilitation and static stretching. J Strength Cond Res 2003;17:489-92.

7 Cornelius WL, Rauschuber MR. The relationship between isometric contaction durations and improvement in acute hip joint flexibility. Journal of Applied Sport Science Research 1987;1:39-41.

8 Schmitt GD, Pelham TW, Holt LE. A comparison of selected protocols during proprioceptive neuromuscular facilitation stretching. Clinical Kinesiology 1999:53:16-21.

9 Holt LE, Pelham TW, Campagna PD. Hemodynamics during a machine-aided flexibility protocol. Can J Appl Physiol 1995;20:407-16.

10 Walter J, Figoni SF, Andres FF, et al. Training intensity and duration in flexibility. Clinical Kinesiology 1996;50:40-5.

11 Magnusson SP, Simonsen EB, Aagaard P, et al. Mechanical and physiological responses to stretching with and without preisometric contraction in human skeletal muscle. Arch Phys Med Rehabil 1996;77:373-8. 